\title{
Adoption of Service Oriented Computing from the IT Professionals' Perspective: An e-Government Case Study
}

\author{
Ilse Baumgartner and Peter Green \\ Business School \\ The University of Queensland, St Lucia QLD 4072, AU \\ I.Baumgartner@business.uq.edu.au \\ P.Green@business.uq.edu.au
}

\begin{abstract}
The case study reported in this paper focuses on the question: what are the critical factors that influence IT professionals' intention to adopt the Service Oriented Computing (SOC) paradigm? This work reports a case study examining the e-Government initiative in a middle-sized European city. It uses an initial SOC adoption model developed through a proceeding interview-based exploratory study [2]. The current study has two principle aims. The first aim is to "shed some light" on the IT professionals" acceptance of such complex technological approaches as Service Oriented Computing in the e-Government sector and to report key learning issues emerging from the case study. The second aim of the case study is to bring further credibility to the first study and to validate empirically its assertions. Some of the major findings of the study are the replacement of the complexity variable by the variable of maintainability, and the replacement of the trust and dependency variables (both of interpersonal rather than of technical nature) by the variable of external involvement. The results of the study also suggest the introduction of the "champion" of the approach variable. Theoretical and practical implications that follow are presented and discussed.
\end{abstract}

Keywords: e-Government; technology acceptance; acceptance of Service Oriented Computing

\section{Introduction}

Presently, public administration systems in many countries of the world are undergoing fundamental restructuring and reorganisation as a result of the emerging e-Government strategies. These new "online-e-Government" services are designed to change radically the experiences that citizens and businesses have in dealing with government. It is not surprising therefore that intensive discussion about the 
most appropriate information systems architectures to deal with the introduction of online-delivered government services is unfolding amongst practitioners and researchers presently [e.g. 5, 12, 21].

Service oriented computing (SOC), together with the fundamental concept of web services, has a high potential to enable and support successfully the evolution of e-Government. While traditional software development approaches focused mainly on individual components, the SOC paradigm emphasises the role of interaction and collaboration defining services as crosscutting system properties. While relying on a set of well-known technologies (XML and HTTP, WSDL, SOAP and UDDI), SOC combines these technologies in a new and innovative way enabling the development of distributed, interoperable and extendable systems and applications. However, the adoption and practical use of the SOC paradigm is usually associated with very diverse problematic issues. What are the financial and technical risks of implementing an SOC-based solution? How will the enormous complexity of an SOC-based system be addressed? What skills are necessary to build and maintain an SOC-based system? How secure will this system be? Are there any reference resources available?

The most significant motivation for this research-in-progress work is the lack of studies examining the adoption of new technologies, techniques, approaches or tools from the perspective of IT professionals. While there is extensive research on technology acceptance from the end-end user perspective only very few studies (e.g. $[1,8,18]$ ) examine and analyse the individual-level acceptance of new technologies, tools or methodologies by IT professionals. In addition to that, almost all of these studies are focusing on the acceptance of technological approaches in commercial settings and the results produced by most of these studies are hardly generalisable to such a multidimensional and complex paradigm as Service Oriented Computing without additional and focused research.

This work argues that an individual decision on whether or not using the SOC paradigm is justified and meaningful in a particular context has to be made by every single IT professional to make an organisational adoption truly successful. Using an e-Government case study, this study examines a number of critical factors that influence the IT professionals' individual-level decision to adopt the Service Oriented Computing paradigm.

\section{Initial research model}

Due to the absence of an established theoretical framework in the domain of individual-level technology acceptance in the IT industry, an exploratory investigation [2] was carried out in a commercial setting to formulate an initial SOC adoption model (see Fig. 1). 


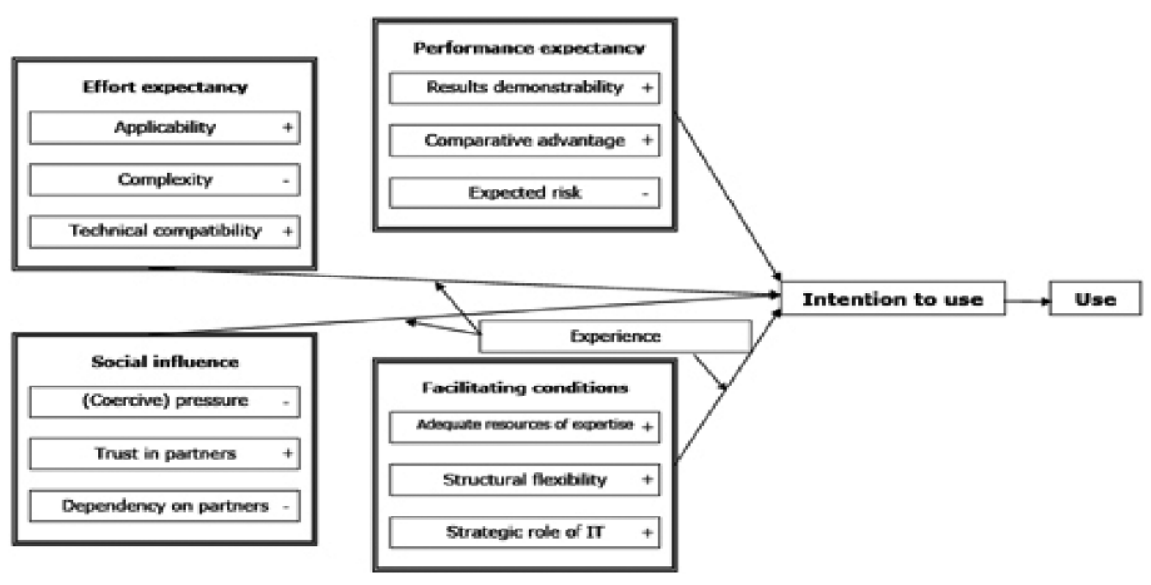

Figure 1. The initial SOC adoption model.

Short description of the constructs of the initial model follows.

\subsection{Facilitating conditions}

In the IS literature, the importance of integrating IT and business strategy has been extensively discussed pointing out that IT affects firm strategies and that strategies have IT implications. The strategic role of IT variable emerges from the connections between IT and the core objectives and propositions of an organisation and is primarily associated with the level of influence senior IT professionals have in an organisation. The higher is the influence level of the IT personnel the higher is the probability of the acceptance of such an approach as SOC.

The structural flexibility variable (having positive influence direction) complements the strategic role of IT variable and refers to the flexibility and efficiency of the IT-related decision-making process in an organisation.

The variable of adequate resources of expertise refers to existing and available external and internal resources (i.e. practical and theoretical knowledge, experience, existing successful/unsuccessful implementation references) which could be useful and supportive when implementing SOC-based systems and solutions. This variable is positively associated with the adoption intentions.

\subsection{Performance expectancy}

Result demonstrability is defined as the tangibility of the results of using the innovation including their observability and communicability [14, p.203]. This variable is positively associated with the intention to adopt the SOC paradigm.

Comparative (relative) advantage is understood as the "degree to which an idea is perceived as being better than the idea it supercedes" [20, p.212], and this variable is negatively associated with the adoption intentions. 
The expected risk variable has negative influence direction and is generally representing technical and business-related risks associated with SOC implementations.

\subsection{Effort expectancy}

Complexity is the "degree to which an innovation is perceived as relatively difficult to understand and use" [20, p.242]. This variable is negatively associated with adoption intentions.

Technical compatibility refers to the degree of compatibility SOC-based solutions and systems are capable of having with existing solutions and applications (including issues such as possibility of incremental implementations, migration of assets, leverage of existing assets which positively contribute to the IT professionals' intentions to adopt the SOC approach). Technical compatibility has positive influence on the adoption intentions.

In the initial SOC adoption model, applicability refers to the extent to which business processes of a specific organisation are suitable to be implemented as "services".

\subsection{Social factors}

Trust and dependency represent two interrelated, but clearly distinguishable variables of interpersonal (rather than technical) nature. While the feeling of having trustworthy partners positively influence the intention of IT professionals to adopt the SOC paradigm, the degree of potential dependency on partners cause exactly the opposite effect.

The (coercive) pressure variable combines two different types of pressure pressure from internal organisational structures and pressure from external entities (e.g. business partners, customers) - and is negatively associated with the intentions of IT professionals to adopt the SOC paradigm.

\section{Moderating variable}

The experience moderating variable refers to the adopter's general IT-related professional experience.

\section{Research methodology}

Our previous exploratory study [2] used interviews with senior IT professionals primarily working in commercial settings to formulate the initial SOC adoption model (Figure 1). In order to enhance the external validity of the emerging SOC adoption model and potentially gather new aspects from another setting, an eGovernment initiative was selected for this case study.

An established case study method proposed by Yin [27] was followed in order to institute rigor in the methodology of this research work. The organisation 
for the case study reported in this paper was selected and the data collection protocol was developed. Due to the nature of the research question explored by the current study and due to a number of restrictions (access restrictions to archival records in a governmental agency, time restrictions regarding observations imposed by the subject organisation on the investigators), we decided to focus on three sources of evidence: documents provided by the subject organisation, focused interviews/informal discussions, and physical artefacts.

The primary data gathering was accomplished using focused interviews and informal discussions (three formal interviews and five informal discussions with senior IT professionals involved in the day-to-day e-Government environment development and support). In addition to that, physical artefacts were examined. The examination of supporting documentation provided by the subject organisation (such as protocols of internal meetings, documentation used in several external meetings and information sessions to present the e-Government initiative and other information) was performed after the interview process.

\section{Data analysis}

The research work used a data analysis method described by Miles and Huberman [13]. As suggested by them, three different activities were undertaken concurrently - data reduction, data display, and conclusion drawing/verification. The data segments resulting from selecting, simplifying, and abstracting the raw case study data were combined, summarised, grouped and then coded into categories derived from the initial theoretical framework of the study. Tables 1 and 2 show the high-level picture resulting from the analysis of the case study data. The detailed analysis leading to these results is explained subsequently.

\begin{tabular}{lll}
\hline Construct & Variables & Results \\
\hline $\begin{array}{l}\text { Facilitating } \\
\text { conditions }\end{array}$ & Adequate resources of expertise $(+)$ & Supported \\
& The strategic role of IT $(+)$ & Supported \\
& Structural flexibility of the organisation $(+)$ Supported \\
Social & (Coercive) pressure $(-)$ & Supported \\
influence & Dependency on partners $(-)$ & $\begin{array}{l}\text { Dependency and trust supported but } \\
\text { mainly with a technical focus }\end{array}$ \\
& Trust in partners $(+)$ & Supported \\
Effort & Applicability $(+)$ & Supported but with a different focus \\
expectancy & Complexity $(-)$ & Supported \\
& Technical compatibility $(+)$ & Supported \\
Performance & Results demonstrability $(+)$ & No evidence available \\
expectancy & Comparative advantage $(+)$ & Supported \\
& Expected risk $(-)$ & \\
\hline
\end{tabular}

Table 1: Constructs and Variables 


\begin{tabular}{lll}
\hline Construct & Moderating variable & Results \\
\hline Facilitating conditions & Experience & Supported \\
Social influence & Experience & Supported \\
Effort expectancy & Experience & Supported \\
Performance expectancy & - & - \\
\hline
\end{tabular}

Table 2: Constructs and moderating variable

\subsection{Facilitating conditions}

All three facilitating condition construct variables (strategic role of IT, adequate resources and structural flexibility) were strongly supported by the case study evidence material.

The advanced state of the project was primarily associated with the strong strategic role of IT and with the exceptionally strong IT leadership. The flat organisational structure and the remarkable mutual trust between the CIO and the leaders of other organisational structures apparently facilitate quick and unbureaucratic IT-related decisions.

Concerning the CIO's position in the subject organisation, one of the interviewed senior IT professionals, for example, noted:

"He [CIO] has an exceptionally strong position here in our organisation."

Regarding the structural flexibility of the organisation the same interviewee stated:

"We have senior management which is very open-minded towards new visions. Decisions are made very quickly."

Concerning the adequate recourses of expertise, all interviewees confirmed the importance of available and accessible external knowledge. They stressed the fact that there are several possibilities to access theoretical knowledge and expertise, however, the most important issue is lack of on-going reference projects enabling the exchange of first-hand practical experiences.

The CIO, for example, noted in this regard:

"There is a lot of [theoretical SOC] knowledge out there, but there are very few productive systems, successful systems, which could be looked at."

\subsection{Social Influence}

While one of the social influence construct variables ((coercive) pressure) was strongly supported by the case study evidence material, the dependency on and trust in partner's variables experienced major revision.

Very interesting insights appeared in relation to the (coercive) pressure variable within the social influence construct. Two different contexts were described by the interviewees: coercive pressure from higher-level government agencies on the subject organisation and coercive pressure on lower-level partners exerted by the subject organisation itself. 
Contrary to the initial theoretical framework of the study both, the dependency variable and the trust variable, were mainly associated with technical rather than with interpersonal issues. As noted by the CIO:

"We use SOC at a very sensible point of the enterprise, namely, for supporting automated communication and transactions. And it is vital to be able to trust the technology that it will work exactly as required, because if it doesn't work you have a major problem."

This effect might, however, be explainable when considering the difference between SOC-based environments implemented in a commercial organisation as opposed to an e-Government environment. In the context of trust and dependency the interviewees clearly confirmed that the level and intensity of "external" involvement will have direct influence on the SOC adoption intentions. This view is, indeed, congruent with the initial theoretical framework of the study - the interpersonal dependency and trust issues will become vital only in case of involvement of "external" and "unknown" partners. In the opposite case (involving only "internal" and "known" people, structures, partners) problems related to interpersonal dependency and trust will diminish radically, and they will not play any major role when considering the adoption of the SOC paradigm.

\subsection{Effort Expectancy}

In the context of the effort expectancy construct two of the variables (applicability and technical compatibility) were supported by the case study data, while the remaining variable of complexity experienced major revision.

The role of complexity was basically supported by the case study evidence material. SOC-based environments are clearly associated with complex implementations based on interrelation, collaboration and dependencies. However, two important issues should be mentioned in this context. First, it was remarkable that this potential complexity was not considered an issue that would have an exclusively negative influence on the intention to adopt and use SOC for building the eGovernment-enabled environment. Second, in the context of this particular study, it became obvious that complexity is considered an "invariable feature" of SOC rather than a problem that has to be discussed and evaluated.

One of the interviewees noted in this context:

"We know that it will get very complex, and we know that there will be many dependencies. But at the very end, through segmentation and refinement, it will-ultimately - become simpler.

Thus, in this context the complexity variable seems to be indirectly replaced by a variable like "maintainability" (i.e. the ease with which a software system can be modified to e.g. correct faults, improve performance, integrate new components or services or adapt to a changed environment).

While applicability (though supported by the case study data) did not represent a major issue for the interviewees, the technical compatibility appeared to have had a very strong influence on the decision to take an SOC-based approach when starting to implement the e-Government system.

As noted by one of the interviewees:

"[It is] a vision how to get the administrative system of our municipality which contains an extremely high number of very heterogeneous applica- 
tions, a highly distributed environment, towards a very strongly integrated solution, at the same time continuing to support this heterogeneity."

As shown by the case study, an e-Government-enabled environment does not exist in isolation. It coexists with many back-office applications that have been built using very different (and older) technologies. It will usually be impossible to rebuild these applications using state-of-the-art technologies and to seamlessly integrate them with the new SOC-based environment. Thus, the IT professionals will have to challenge the capability of SOC to enable integration of existing and very heterogeneous systems and applications. This issue (i.e. integration of existing - and extremely heterogeneous - systems and applications, migration of assets, leverage of existing assets) was also strongly supported by the documentation (especially architecture-related documentation) made available by the subject organisation.

\subsection{Performance Expectancy}

Finally, performance expectancy represents the least discussed construct of the theoretical framework of this study.

As far as the comparative advantage variable is concerned, none of the interviewees mentioned any other approach considered when discussing the basic architectural and technical implementation direction of the new e-Government initiative. Moreover, none of the interviewees mentioned any attempt to compare SOC with older and more conventional approaches, or to determine the advantages SOC has compared to conventional approaches.

As far as the results demonstrability variable is concerned, the most important issue discussed by the interviewees was the ability to demonstrate the progress of the SOC-based e-Government project to external interested parties (e.g. journalists, other government agencies) rather than to internal stakeholders.

The expected risk variable appears to have had substantial impact on the adoption decision process; however, similar to the complexity variable, this variable did not necessarily have an exclusively negative impact on the decision whether or not to adopt SOC. While admitting that the expected risk (technical as well as interpersonal) might be considerably high the interviewees regarded it both, a detriment as well as a challenge.

\section{Summary and the refinement of the SOC adoption model}

The case study evidence material does not suggest any changes to the existing three variables of the facilitating conditions construct (i.e. adequate resources of expertise, structural flexibility of the organisation, and the strategic role of IT in an organisation) and confirms the influence of the facilitating conditions construct on the intention to adopt the SOC paradigm. Additionally, the data analysis confirms the positive direction of influence of all three variables constituting the facilitating conditions construct.

In addition to the three existing variables for this construct, the data analysis suggests that a fourth variable should be added to the facilitating conditions con- 
struct - strong IT leader as a "champion" of the approach [16] (having strong positive influence). The role of a "champion" of an approach has been described by a large number of studies [e.g. 16, 17, 8]. As Palvia and Chervany note: "[ $[.$.$] the$ role of the project champion cannot be understated. In all stages this experimental factor produced the largest gains in the predictions that the system will succeed" $[16, p$ 52]. Neither the strategic role of IT variable nor the structural flexibility variable cover the effect that derives from strong and influential IT leadership in an organisation. As shown by the current case, even considerable structural flexibility in the organisation and a strong strategic role of IT would not have been considered sufficient for taking an SOC-based approach if a strong and goaloriented IT leadership - a "champion" of the approach - was missing.

As far as the effort expectancy construct is concerned, the current case study confirms the influence of this construct on the adoption intention. For two of the constituent variables, technical compatibility and applicability, the positive influence direction was clearly supported. The third variable, complexity - although discussed in extenso - did not show a clear influence direction. Moreover, the data analysis suggests that complexity is rather considered an "invariable" feature of SOC than an issue that has to be discussed and dealt with when deciding upon the adoption of SOC. Therefore, the variable of maintainability (having positive influence direction) replaces the variable of complexity in the refined SOC adoption model.

Within the performance expectancy construct, the influence of two variables - results demonstrability (positive) and expected risk - was confirmed by the data of the case study. Both constituent variables will be retained in the refined SOC adoption model with the same influence direction. Contrary to that, the influence of the comparative advantage variable could not be confirmed by the study as no data supporting the influence of this variable emerged from the case study. However, based on existing research in the field of technology acceptance in the IT industry, it can be assumed that the comparative (i.e. relative) advantage variable will have a certain impact on innovation adoptions, at least in commercial environments where the issues of investments and financial risk definitely have more severe impact than in public administration settings. (In a commercial environment, comparing existing conventional (and functioning) approaches with such an approach as SOC that requires a massive financial investment will most probably be a serious issue and will consequently influence the adoption decision). Based on this assumption, the comparative advantage variable will be retained in the refined SOC adoption model with the same influence direction.

The social influence construct has also been modified to a certain extent. The (coercive) pressure variable was clearly supported by the case study evidence material, and, thus, will be retained in the refined SOC adoption model with the same influence direction. The role of the trust and dependency variables (both being of interpersonal nature) seems to be partially neutralised by the effect of the (coercive) pressure variable, the issue of standardisation (which, to a considerable extent, is a result of coercive pressure), and the nature of the project environment (public administration/e-Government rather than commercial). However, the importance of avoiding or minimising "external" involvement in SOC-based solutions is strongly supported by the current study. Thus, both variables, interpersonal 
dependency and interpersonal trust, will be replaced by a single variable reflecting this issue - external involvement (having a negative influence direction).

Considering the above arguments as a whole, Figure 2 represents the refined SOC adoption model:

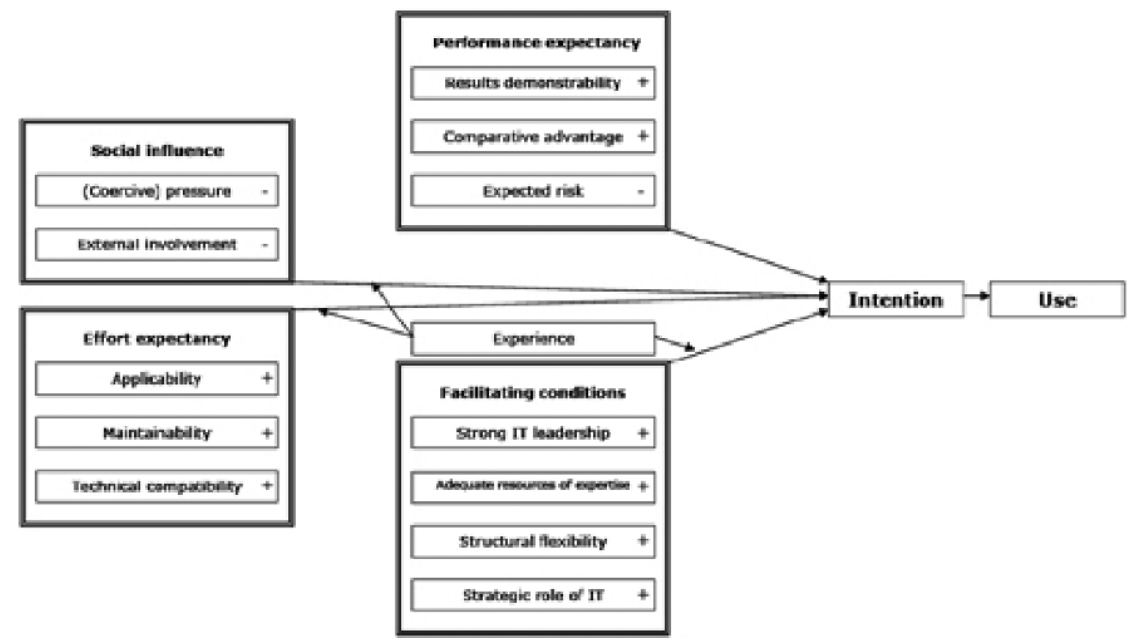

Figure 2. Refined SOC adoption model.

\section{Conclusion, Limitations, and Further Work}

In general, this study represents a step toward a better understanding of the adoption process of such complex innovations as Service Oriented Computing. The current study focuses on the individual-level perspective of IT professionals using this particular approach to support introduction and development of eGovernment-enabled services.

The theoretical framework of this research work was initially developed through an exploratory interview-based study [2]. While the four major constructs exposed by this initial theoretical framework were confirmed the current study suggested several changes.

It is important to critically evaluate the study and its results. The present study has certain limitations that need to be taken into account when considering its contributions.

The refinement of the model is being performed using data from only one case. While every effort was taken to mitigate against selection bias in the subject organisation, the knowledge obtained from this study is necessarily bounded by the characteristics of the organisation and the characteristics of the interviewees. However, the e-Government initiative implemented by the selected subject organisation can be considered an exemplar and successful application of the SOC paradigm, the subject organisation plays a unique role in the Austrian e-Government standardisation process and the interviewed IT professionals have substantial work 
experience with SOC.

A concern with all qualitative inquiry methods (including interview-based case studies) is the propensity for the introduction of bias (e.g., researcher bias and expectation bias) into the data gathering and analysis stages. During the interview process, document and artefact examination, and the data analysis, every care was taken not to allow research biases to affect the direction of the interview, the observations recorded, and the analysis attained. In addition to that aspect, two of the three remedies proposed by Yin were used: multiple sources of evidence, and the revision of the basic structure of the case study report by the key informants.

In subsequent work, the refined SOC adoption model that has been presented in this paper will be validated using a survey instrument. Based on the results of this paper as well as on the results of the preceding study [2], a survey instrument will be developed, pilot-tested and then web-distributed to European IT experts who are utilising the Service Oriented Computing approach in their professional work or presently considering using it.

While carrying out the last validation step, it will be necessary to consider several additional aspects in order to enhance the quality of the results.

First, when collecting the respondents' demographic data it will be necessary to pay special attention to the type of the company the respondent is working for (i.e. commercial or public administration type of organisation). Second, it will be necessary to distinguish between two types of experience - experience with Service Oriented Computing and experience in the IT industry in general. Third, regarding the dependent variable, intention to use, it will be important to distinguish between the intention to start using the Service Oriented Computing paradigm and the intention to continue using it.

Ultimately, we expect to gain more knowledge of the determinants of IT professionals' intentions to adopt the Service Oriented Computing paradigm.

\section{References}

[1] R.Agarwal, J. Prasad, A field study of the adoption of software process innovations by information systems professionals, IEEE Transactions on Engineering Management 47:3 (2000) 295-308

[2] I.Baumgartner, P.Green, Service Oriented Computing: what do IT professionals think?, (2007) (currently under review)

[3] L. Carter, F. Belanger, The Influence of Perceived Characteristics of Innovating on eGovernment Adoption, Electronic Journal of e-Government 2:1 (2003) 11-20

[4] J. D'Ambra, W. Huang, V. Bhalla, An empirical investigation of the adoption of eGovernment by Australian citizens: some unexpected research findings, Journal of Computer Information Systems 3:1 (2002) 15 - 22

[5] Z. Ebrahim, Z. Irani, E-Government adoption: architecture and barriers, Business Process Management Journal 11:5 (2005) 589-611

[6] M. J. Gallivan, Organizational adoption and assimilation of complex technological innovations: development and application of a new framework, SIGMIS Database Special issue: Adoption, diffusion, and infusion of IT 32: 3 (2001) 51-85

[7] D. Gilbert, P. Balestrini, D. Littleboy, Barriers and benefits in the adoption of e-Government, The International Journal of Public Sector Management 17:4 (2004) 286-301

[8] R. A. Johnson, B. C. Hardgrave and, E. R. Doke, An industry analysis of developer beliefs about object-oriented systems development, SIGMIS Database 30:1 (1999) 47-64 
[9] M. Khalifa, J.M. Verner, Drivers for software development method usage, IEEE Transactions on Engineering Management 47:3 (2000) 360-369

[10] B. Kitchenham, L. Pickard, S. L. Pfleeger, Case Studies for Method and Tool Evaluation, IEEE Software 12:4 (1995) 52-62

[11] A. Leigh, R. Atkinson, Breaking Down Bureaucratic Barriers: The Next Phase of Digital Government, Progressive Policy Institute, Technology \& New Economy Project (2001)

[12] F. Li, Implementing E-Government strategy in Scotland: current situation and emerging issues, Journal of Electronic Commerce in Organisations 1:2 (2003) 44-65

[13] M.B. Miles, A.M. Humberman Qualitative Data Anaysis: A sourcebook of new methods, Sages Publications, Newsbury Park (1984)

[14] G.C. Moore, I.Benbasat, Development of an instrument to measure the perceptions of adopting an information technology innovation,. Information Systems Research 2:3 (1991) 192222

[15] W.J. Orlikowski, D.C. Gash, Technological Frames: Making Sense of Information Technology in Organizations, ACM Transactions on Information Systems 2:2 (1994) 174-207

[16] S.C. Palvia, N.L. Chervany, An experimental investigation of factors influencing predicted success in DSS implementation, Information and Management 29:1 (1995) 43-53

[17] S.C. Palvia, N.L. Chervany, Effect of environmental and process variables on DSS implementation success: evidence from an experimental investigation for the moving stage of change, Decision Support and Knowledge Based Systems Track 3 (1989) 812-21

[18] C. K. Riemenschneider, B. C. Hardgrave, and F. D. Davis, Explaining Software Developer Acceptance of Methodologies: A Comparison of Five Theoretical Models, IEEE Transactions on Software Engineering 28:12 (2002) 1135-1145

[19] T.L. Roberts, M.L. Gibson, Jr., K.T. Fields, R.K. Rainer, Jr, Factors that impact implementing a system development methodology, IEEE Transactions on Software Engineering, 24:8 (1998) 640-649

[20] E.M. Rogers, Diffusion of Innovations (The Free Press, New York, 1995, 4th ed.)

[21] S. Smith, R. Jamieson, Determining key factors in e-Government information system security, Information Systems Management. Boston 23:2 (2006) 23-33

[22] R. Stake, The art of case research. Thousand Oaks, CA: Sage Publications (1995)

[23] F. Sultan, L. Chan, The adoption of new technology: the case of object-oriented computing in software companies, IEEE Transactions on Engineering Management 47:1 (2000) 106-126

[24] V. Venkatesh, M. G. Morris, G. B. Davis, and F. D. Davis, User Acceptance of Information Technology: Toward a Unified View, MIS Quarterly 27:3 (2003) 425-478

[25] M. Warkentin, D. Gefen, P. Pavlou, G. Rose, Encouraging Citizen Adoption of eGovernment by Building Trust, Electronic Markets 12:3 (2002) 157-162

[26] X. Wei, J. Zhao, Citizens' requirement analysis in Chinese e-Government, Proceedings of the 7th international Conference on Electronic Commerce 113 (2005) 525 - 428

[27] R. Yin, Case study research: Design and methods, Beverly Hills, CA: Sage Publishing 2nd ed. (1994)

[28] M.V. Zelkowitz, D.R. Wallace, Experimental Models for Validating Technology, IEEE Computer 31:5 (1998) 23-31

[29] 2006 Online Availability of Public Services: How is Europe Progressing?, full Capgemini report available on http://www.capgemini.com/resources/thought_leadership /2006_online_availability_of_public_services/ (last access September 6, 2007)

[30] Platform "Digitales Österreich" (Digital Austria), available on http://reference.eGovernment.gv.at/Digitales_OEsterreich.725.0.html (last access September 6, 2007)

\section{Interview Questions}

Have you ever experienced any type of pressure regarding the architectural and technical implementation direction of the new e-Government environment? If yes, would you describe this pressure? 
What role, in your personal opinion, did the individual members of the IT department in general and the leader of the IT department in particular play when deciding upon the architectural and technical implementation direction of the new e-Government environment?

How did you acquire the technical and architectural SOC-related knowledge necessary to realise this particular project? Did you experience the need to consult external resources of SOC expertise in the course of the new e-Government project?

Did you experience any external control concerning the results of the new SOC-based eGovernment initiative? Was it necessary - at any stage of the project - to prove the actual status/results of the project to external or internal stakeholders?

Was SOC the only approach you personally considered when arguing about the architectural and technical implementation direction of the new e-Government environment?

Did you consider any particular risks?

What issues would you associate with interpersonal trust and dependency in the context of the new SOC-based e-Government initiative?

What would you consider the most important problematic issues of the SOC approach?

What would you personally consider the most important advantages of the SOC approach?

Was the heterogeneity of the present environment an issue when deciding upon the architectural and technical implementation direction of the new e-Government environment?

Considering the present environment, do you think that all major business processes will be integrated into the new environment as "services"? 